\title{
Anxiety and Depression in Physical Therapists during the COVID-19 Pandemic: A Cross-sectional Survey in Gwangju, South Korea
}

\author{
Se-Ju Park, PT, PhD • Ho-Jin Jeong, PT, PhD • Byeong-Geun Kim, PT, MS ${ }^{\dagger}$
}

Department of Physical Therapy, Nambu University

Received: March 8, 2021 / Revised: March 12, 2021 / Accepted: April 15, 2021

(c) 2021 J Korean Soc Phys Med

\begin{abstract}
| Abstract |
PURPOSE: Physical therapists are at risk of becoming infected because they are in contact with or within 2 meters of the patient while treating them. The purpose of this study is to investigate the anxiety and depression of physical therapists during the coronavirus disease 2019 (COVID-19) pandemic and to identify the factors that affect anxiety and depression.
\end{abstract}

METHODS: A cross-sectional survey was conducted during the pandemic. The survey were completed by 84 physical therapists in 24 local hospitals in Gwangju, South Korea. The Generalized Anxiety Disorder-7 (GAD-7) scale and the Patient Health Questionnaire-9 (PHQ-9) were used to evaluate their anxiety and depression status. Logistic regression models were used to identify the general characteristics factors related to anxiety or depression.

RESULTS: It was found that $40(47.6 \%)$ respondents had anxiety, and 37 (44\%) had depression. If there was a child under the age of seven in the physical therapist's family, a high probability of anxiety $(\mathrm{p}<.05)$ prevailed. Physical therapists

$\dagger$ Corresponding Author : Byeong-Geun Kim qudrms_92@naver.com, https://orcid.org/0000-0002-7358-7389 This is an Open Access article distributed under the terms of the Creative Commons Attribution Non-Commercial License (http://creativecommons.org/licenses/by-nc/3.0) which permits unrestricted non-commercial use, distribution, and reproduction in any medium, provided the original work is properly cited. in their 30s had a higher risk of depression than those in their $40 \mathrm{~s}(\mathrm{p}<.05)$. The risk of anxiety and depression in physical therapists who were with patients who did not wear masks was higher than for those who were with patients who wore masks $(\mathrm{p}<.05)$.

CONCLUSION: The anxiety and depression status of physical therapists in Gwangju, South Korea, during the pandemic of COVID-19 was poor. It is necessary to wear a mask for all people during physical therapy.

Key Words: Anxiety, Covid-19, Depression, Mental health, Physical therapists

\section{Introduction}

The outbreak of coronavirus disease 2019 (COVID-19) began in Hubei Province, China, at the end of December 2019 [1], and has spread all over the world; many people have been infected or died from this deadly disease [2,3]. It was officially referred to as COVID-19 by the world health organization (WHO) in February 2020, and the first case in South Korea was reported on January 20, 2020. South Korea has implemented a wide range of national response strategies, including extensive tracking, virus testing, isolation, and social distancing to reduce the spread of the virus $[4,5]$. However, the number of confirmed cases continues to increase due to local infection that despite 
the efforts of the government and the people. In this case, medical workers working in hospitals are adversely affected by physical and mental health.

Several studies on mental health in medical workers have been conducted previously. High levels of stress, anxiety, and depression for medical workers working in hospitals were reported for severe acute respiratory syndrome (SARS) in 2003 and middle east respiratory syndrome (MERS) in 2015 [6-8]. Recent COVID-19 research has demonstrated that medical workers experienced more problems such as insomnia, anxiety, and depression than non-medical workers [9]. Following the outbreak of COVID-19, many studies have been conducted on the mental health of physicians and nurses [10-13]. Most of previous studies were medical workers of directly related to COVID-19. Therefore, studies for other medical workers are shortage. Among medical workers, physical therapists do treatment for patients during direct contact with patients in hospitals. Also, patients visit hospitals for physical therapy during the COVID-19 pandemic.

COVID-19 is highly contagious and differs from other viruses as it has an incubation period of approximately two to ten days before symptoms begin to manifest following person-to-person transmission [14,15]. Aerosol particles with COVID-19 generated from coughing or sneezing can survive in the air for more than three hours, and people can become infected with the virus when it is inhaled or enters the mucous membranes of the eye [16]. When an infected person coughs or sneezes, the particles fall within 2 meters [17]. Therefore, When treating a patient, physical therapists face the risk of coming in contact with the infected person. If a patient visits the hospital and receives physical therapy during the incubation period, the physical therapist may also be infected. The purpose of this study is to investigate the anxiety and depression of physical therapists during the COVID-19 pandemic and to identify the factors that affect anxiety and depression.

\section{Methods}

\section{Participants}

This study was a cross-sectional survey study conducted in Gwangju, South Korea. It was approved by the Ethics Committee of the Nambu University (IRB1041478-2020HR-026). The sample size was analyzed using the G-power 3.1 program. The effect size was .5, the significance level was .05 , the power was $95 \%$, and the degrees of freedom were 6 . The minimum number of samples was 84 . This study used convenience sampling strategy. Considering the high infectivity of COVID-19, the supply rate of smartphones in South Korea, and the feasibility of electronic questionnaires, an online questionnaire powered by Google was used for answering the paperless questionnaire. We surveyed 24 local hospitals from October 5. The research objects of this study were 84 physical therapists in 24 local hospitals. Those participants who had a physical therapist license and worked in a hospital in Gwangju were included in this study. Physical therapists who have been diagnosed with mental health-related diseases before COVID-19 and those who are currently taking mental illness-related drugs were excluded from the study.

\section{Survey}

The survey began with the informed consent of all participants and considered the general characteristics, anxiety, and depression faced by them. The general characteristics recorded personal information, including age range, gender, family members, clinical experience, treatment type, patient type, treatment room type, and if the patient were wearing a mask. Anxiety was measured with the Generalized Anxiety Disorder-7 (GAD-7) scale developed by Spitzer [18]. GAD-7 is a seven-item anxiety scale with total scores ranging from 0 to 21 . GAD-7 is a scale with high reliability, and a total score of five or higher indicates anxiety [19]. Depression was measured with the Patient Health Questionnaire-9 (PHQ-9) developed by Kroenke [20]. PHQ-9 is a nine-item depression scale 
Table 1. Association of demographic data of physical therapists with the presence of anxiety

\begin{tabular}{|c|c|c|c|c|}
\hline Variable & Total & Anxiety & Non-Anxiety & $\mathrm{p}$ \\
\hline Total, n (\%) & $84(100)$ & $40(47.6)$ & $44(52.4)$ & \\
\hline \multicolumn{5}{|c|}{ Age range } \\
\hline $20 \mathrm{~s}$ & 57 & 25 & 32 & \multirow{3}{*}{.446} \\
\hline $30 \mathrm{~s}$ & 20 & 12 & 8 & \\
\hline $40 \mathrm{~s}$ & 7 & 3 & 4 & \\
\hline \multicolumn{5}{|c|}{ Sex } \\
\hline Male & 35 & 16 & 19 & \multirow{2}{*}{.768} \\
\hline Female & 49 & 24 & 25 & \\
\hline \multicolumn{5}{|c|}{ Family member } \\
\hline Alone & 22 & 11 & 11 & \multirow{4}{*}{$.037^{*}$} \\
\hline$\leq 7$ year-old in family & 9 & 8 & 1 & \\
\hline 8-64 year-old in family & 50 & 19 & 31 & \\
\hline$\geq 65$ year-old in family & 3 & 2 & 1 & \\
\hline \multicolumn{5}{|c|}{ Clinical experience } \\
\hline $1-4$ years & 45 & 22 & 23 & \multirow{3}{*}{.565} \\
\hline 5-9 years & 31 & 13 & 18 & \\
\hline $10<$ years & 8 & 5 & 3 & \\
\hline \multicolumn{5}{|c|}{ Treatment type } \\
\hline Nervous system & 42 & 22 & 20 & \multirow{2}{*}{.382} \\
\hline Musculoskeletal system & 42 & 18 & 24 & \\
\hline \multicolumn{5}{|c|}{ Patient type } \\
\hline Inpatient & 13 & 6 & 7 & \multirow{3}{*}{.235} \\
\hline Outpatient & 3 & 0 & 3 & \\
\hline In + Outpatient & 68 & 34 & 34 & \\
\hline \multicolumn{5}{|c|}{ Treatment room type } \\
\hline Personal treatment room & 16 & 9 & 7 & \multirow{2}{*}{.442} \\
\hline Communal treatment room & 68 & 31 & 37 & \\
\hline \multicolumn{5}{|c|}{ Patient wearing a mask } \\
\hline Yes & 76 & 33 & 43 & \multirow{2}{*}{$.018^{*}$} \\
\hline No & 8 & 7 & 1 & \\
\hline
\end{tabular}

$* \mathrm{p}<.05$.

with total scores ranging from 0 to 27 . PHQ-9 is a scale with high reliability, and a total score of five or higher indicates depression [21].

\section{Statistical analysis}

All statistical analyses were conducted using SPSS version 25.0 (IBM Corp., Armonk, NY, USA). Anxiety and depression were identified according to general characteristics. A chi-square test was used to confirm the correlation between general characteristics, anxiety, and depression. An additional logistic regression was performed for the relevant variables. The statistical significance level was set to .05 .

\section{Results}

This study surveyed 84 physical therapists from 24 local hospitals. Anxiety was found in $40(47.6 \%)$ of the 
Table 2. Association of Demographic Data of Physical Therapists with the Presence of Depression

\begin{tabular}{|c|c|c|c|c|}
\hline Variable & Total & Depression & Non-Depression & $\mathrm{p}$ \\
\hline Total, n (\%) & $84(100)$ & $37(44)$ & $47(56)$ & \\
\hline \multicolumn{5}{|c|}{ Age range } \\
\hline $20 \mathrm{~s}$ & 57 & 23 & 34 & \multirow{3}{*}{$.041^{*}$} \\
\hline $30 \mathrm{~s}$ & 20 & 13 & 7 & \\
\hline $40 \mathrm{~s}$ & 7 & 1 & 6 & \\
\hline \multicolumn{5}{|c|}{ Sex } \\
\hline Male & 35 & 15 & 20 & \multirow{2}{*}{.853} \\
\hline Female & 49 & 22 & 27 & \\
\hline \multicolumn{5}{|c|}{ Family member } \\
\hline Alone & 22 & 9 & 13 & \multirow{4}{*}{.536} \\
\hline$\leq 7$ year-old in family & 9 & 6 & 3 & \\
\hline 8-64 year-old in family & 50 & 21 & 29 & \\
\hline$\geq 65$ year-old in family & 3 & 1 & 2 & \\
\hline \multicolumn{5}{|c|}{ Clinical experience } \\
\hline $1-4$ years & 45 & 16 & 29 & \multirow{3}{*}{.140} \\
\hline $5-9$ years & 31 & 18 & 13 & \\
\hline $10<$ years & 8 & 3 & 5 & \\
\hline \multicolumn{5}{|c|}{ Treatment type } \\
\hline Nervous system & 42 & 18 & 24 & \multirow{2}{*}{.826} \\
\hline Musculoskeletal system & 42 & 19 & 23 & \\
\hline \multicolumn{5}{|c|}{ Patient type } \\
\hline Inpatient & 13 & 5 & 8 & \multirow{3}{*}{.250} \\
\hline Outpatient & 3 & 0 & 3 & \\
\hline In + Outpatient & 68 & 32 & 36 & \\
\hline \multicolumn{5}{|c|}{ Treatment room type } \\
\hline Personal treatment room & 16 & 10 & 6 & \multirow{2}{*}{.098} \\
\hline Communal treatment room & 68 & 27 & 41 & \\
\hline \multicolumn{5}{|c|}{ Patient wearing a mask } \\
\hline Yes & 76 & 30 & 46 & \multirow{2}{*}{$.009^{*}$} \\
\hline No & 8 & 7 & 1 & \\
\hline
\end{tabular}

$* \mathrm{p}<.05$.

participants, and depression in 37 (44\%) (Tables 1 and 2).

There was a significant association between anxiety and the general characteristics of physical therapists (family members, patients wearing masks $)(p<.05)(* \mathrm{p}<.05$. Table 1). There was a significant relationship between depression and the general characteristics of the physical therapists (age range, patients wearing masks) $(\mathrm{p}<.05)$ (Table 2).

Related general trait variables and their effects on anxiety and depression were further identified. The risk of anxiety in physical therapists who were residing with family members, including children under seven years, was 13.053 times higher than for those with family members aged 8 to $64(\mathrm{p}<.05)$ (Table 3$)$. The risk of depression in physical therapists who were in their 30s was 11.143 times higher than for those who were in their 40s (p < .05) (Table 4). The risk of anxiety and depression in physical therapists who visited patients who did not wear 
Table 3. Binary Logistic Regression Analysis for the Risk Factor of Family Members' Age-associated with Anxiety

\begin{tabular}{cccc}
\hline Comparison & OR & 95\% CI & $\mathrm{p}$ \\
\hline 8-64 year-old in family vs. Alone & 1.632 & $.593-4.490$ & .343 \\
$8-64$ year-old with family vs. $\leq 7$ year-old with family & 13.053 & $1.512-112.714$ & $.020^{*}$ \\
8-64 year-old with family vs. $\geq 65$ year-old with family & 3.263 & $.277-38.480$ & .348 \\
\hline
\end{tabular}

$* p<.05 ; \mathrm{OR}$, odds ratio; CI, confidence interval.

Table 4. Binary Logistic Regression Analysis for the Risk Factor of Age Range Associated with Depression

\begin{tabular}{ccccc}
\hline Comparison & OR & 95\% CI & $\mathrm{p}$ \\
\hline $40 \mathrm{~s}$ vs. $20 \mathrm{~s}$ & 4.059 & $.458-35.982$ & .208 \\
$40 \mathrm{~s}$ vs. $30 \mathrm{~s}$ & 11.143 & $1.108-112.012$ & $.041^{*}$ \\
\hline
\end{tabular}

$* p<.05 ; \mathrm{OR}$, odds ratio; CI, confidence interval.

Table 5. Binary Logistic Regression Analysis for the Risk Factor of Patients Wearing a Mask Associated with Anxiety.

\begin{tabular}{cccc}
\hline Comparison & OR & $95 \%$ CI & $\mathrm{p}$ \\
\hline Patient wearing a mask, Yes vs. No & 9.121 & $1.069-77.822$ & $.043^{*}$ \\
\hline
\end{tabular}

$* p<.05 ;$ OR, odds ratio; CI, confidence interval.

Table 6. Binary Logistic Regression Analysis for the Risk Factor of Patients Wearing a Mask Associated with Depression

\begin{tabular}{ccccc}
\hline Comparison & OR & $95 \%$ CI & $\mathrm{p}$ \\
\hline Patient wearing a mask, Yes vs. No & 10.733 & $1.256-91.701$ & $.030^{*}$ \\
\hline
\end{tabular}

$* p<.05 ; \mathrm{OR}$, odds ratio; CI, confidence interval.

masks was 9.121 and 10.733 times higher, respectively, than for those with patients who did wear masks ( $p<$ .05 ) (Tables 5 and 6).

\section{Discussion}

The COVID-19 pandemic has caused high levels of stress on physical therapists. This study aimed to assess physical therapists' anxiety and depression status during this period. The study found that physical therapists had $47.6 \%$ anxiety and $44 \%$ depression. In a preceding study reported in April 2020, the rates of anxiety and depression present in physical therapists were $32.3 \%$ and $18.5 \%$ [22], respectively, and the results of this study demonstrated a higher prevalence. The reason why anxiety and depression among physical therapists appeared higher in this study than in previous studies is thought to be due to the prolonged exposure to the virus in the medical field as the pandemic persisted. Another study reported that it was necessary to adjust the working conditions and intensity, both flexibly and systematically, according to the mental health status of medical workers with prolonged exposure to COVID-19 [23].

The study found that among the general characteristics of physical therapists, anxiety was related to family members. The risk of anxiety in physical therapists who had family members under seven years was 13.053 times 
higher than for those with family members aged 8 to 64 . In previous studies, the prevalence of anxiety was 6.727 times higher with family members under six years than those who were alone [22]. Therefore, it was found that physical therapists with families of children under the age of six to seven were prone to higher anxiety. This is because COVID-19 can be transmitted to children by adults, which increases their susceptibility to contracting the disease from their family members who are physical therapists [24]. In South Korea, patients with confirmed COVID-19 are quarantined and are in self-isolation for two weeks after testing. Similarly, in children, self-isolation and a quarantine for two weeks are suggested [25]. Therefore, physical therapists who have families with children are likely to feel more anxious.

This study found that among the general characteristics of physical therapists, depression was related to age range. The risk of depression in physical therapists who were in their 30s was 11.143 times higher than those in their 40s. In previous studies, the prevalence of depression was higher for those in their 30s and 50s than 20s [22]. Prior studies indicated that during the COVID-19 pandemic, the prevalence rate of depression was higher for those in their 30 s than in their 40 s, and that of older people was lower [26]. There are still conflicting results for age and depression during the COVID-19 pandemic. Thus, further research is necessary for this sector to arrive at a stable conclusion.

The present study found that among the general characteristics of physical therapists, anxiety and depression were related to patients' mask use. The risk of anxiety and depression in physical therapists who came in contact with patients who did not wear masks was 9.121 times and 10.733 times higher, respectively, than for those with patients who wore masks. Wearing masks is legally mandatory in South Korea. Therefore, additional confirmation was made for those who did not wear masks. Patients answered that they wore masks at first; however, there were instances where they removed masks when lying on a bed or when breathing was uncomfortable during exercise therapy. Moreover, according to recent studies, it was reported that a surgical or cloth mask that is easy to breathe does not have a detrimental effect on the body when exercising [27]. It is necessary to consider effective methods such as changing patients' mask to one that is more breathable or modifying the treatment method by understanding the patient's condition and treatment plan in advance when the patient is receiving treatment.

There are, however, several limitations to this study. Hospital workers in only one city in South Korea were surveyed for this study. The study did not employ a control group or longitudinal follow-up. The work stress of physical therapists was not considered. Therefore, further research is needed to supplement these limitations.

\section{Conclusion}

The COVID-19 pandemic has resulted in many physical therapists experiencing a high risk of anxiety and depression. Currently, it is mandatory to wear a mask, but there are exceptions. However, it is necessary to wear a mask for all people during physical therapy. The mental health of physical therapists requires constant monitoring. A great deal of attention and improvement is needed so that physical therapists can work in a safe environment.

\section{References}

[1] Huang C, Wang Y, Li X, et al. Clinical features of patients infected with 2019 novel coronavirus in Wuhan, China. Lancet. 2020;395(10223):497-506.

[2] Wiersinga WJ, Rhodes A, Cheng AC, et al. Pathophysiology, transmission, diagnosis, and treatment of coronavirus disease 2019 (COVID-19): a review. JAMA. 2020;324(8):782-93.

[3] COVID-19 Dashboard by the Center for Systems Science 
and Engineering at Johns Hopkins University. COVID-19. https://coronavirus.jhu.edu/map.html. Accessed 18 Nov 2020.

[4] Shim E, Tariq A, Choi W, et al. Transmission potential and severity of COVID-19 in South Korea. Int J Infect Dis. 2020;93:339-44.

[5] Cho SI. Academic community's efforts to guide the fight against coronavirus disease 2019 (COVID-19) epidemic in Korea. J Prev Med Public Health. 2020;53(2):65-6.

[6] Lee AM, Wong JG, McAlonan GM, et al. Stress and psychological distress among SARS survivors 1 year after the outbreak. Can J Psychiatry. 2007;52(4):233-40.

[7] Wu P, Fang Y, Guan Z, et al. The psychological impact of the SARS epidemic on hospital employees in China: exposure, risk perception, and altruistic acceptance of risk. Can J Psychiatry. 2009;54(5):302-11.

[8] Lee SM, Kang WS, Cho AR, et al. Psychological impact of the 2015 MERS outbreak on hospital workers and quarantined hemodialysis patients. Compr Psychiatry. 2018;87:123-7.

[9] Zhang WR, Wang K, Yin L, et al. Mental health and psychosocial problems of medical health workers during the COVID-19 epidemic in China. Psychother Psychosom. 2020;89(4):242-50.

[10] Li Q, Chen J, Xu G, et al. The psychological health status of healthcare workers during the COVID-19 outbreak: a cross-sectional survey study in Guangdong, China. Front Public Health. 2020;8:562885.

[11] Magnavita N, Soave PM, Ricciardi W, et al. Occupational stress and mental health among anesthetists during the COVID-19 pandemic. Int J Environ Res Public Health. 2020;17(21):8245.

[12] Mira JJ, Carrillo I, Guilabert M, et al. Acute stress of the healthcare workforce during the COVID-19 pandemic evolution: a cross-sectional study in Spain. BMJ Open. 2020;10(11):e042555.

[13] Hu N, Li Y, He SS, et al. Impact of the family environment on the emotional state of medical staff during the
COVID-19 outbreak: the mediating effect of self-efficacy. Front Psychol. 2020;11:576515.

[14] Sohrabi C, Alsafi Z, O’Neill N, et al. World health organization declares global emergency: A review of the 2019 novel coronavirus (COVID-19). Int J Surg. 2020;76:71-6.

[15] Guan WJ, Ni ZY, Hu Y, et al. Clinical characteristics of coronavirus disease 2019 in China. N Engl J Med. 2020;382(18):1708-20.

[16] Van Doremalen N, Bushmaker T, Morris DH, et al. Aerosol and surface stability of SARS-CoV-2 as compared with SARS-CoV-1. N Engl J Med. 2020;382(16):1564-7.

[17] Chu DK, Akl EA, Duda S, et al. Physical distancing, face masks, and eye protection to prevent person-to-person transmission of SARS-CoV-2 and COVID-19: a systematic review and meta-analysis. Lancet. 2020;395 (10242):1973-87.

[18] Spitzer RL, Kroenke K, Williams JB, et al. A brief measure for assessing generalized anxiety disorder: the GAD-7. Arch Intern Med. 2006;166(10):1092-7.

[19] Lee SH, Shin C, Kim H, et al. Validation of the Korean version of the generalized anxiety disorder 7 self-rating scale. Asia Pac Psychiatry. 2020;e12421.

[20] Kroenke K, Spitzer RL, Williams JB. The PHQ9: validity of a brief depression severity measure. J Gen Intern Med. 2001;16(9):606-13.

[21] Maroufizadeh S, Omani-Samani R, Almasi-Hashiani A, et al. The reliability and validity of the Patient Health Questionnaire-9 (PHQ-9) and PHQ-2 in patients with infertility. Reprod Health. 2019;16(1):137.

[22] Yang S, Kwak SG, Ko EJ, et al. The mental health burden of the COVID-19 pandemic on physical therapists. Int J Environ Res Public Health. 2020;17(10):3723.

[23] Zhang Y, Xie S, Wang P, et al. Factors influencing mental health of medical workers during the COVID-19 outbreak. Front Public Health. 2020;8:491.

[24] Cao Q, Chen YC, Chen CL, et al. SARS-CoV-2 infection in children: Transmission dynamics and clinical 
characteristics. J Formos Med Assoc. 2020;119(3):670-3.

[25] Park JY, Han MS, Park KU, et al. First pediatric case of coronavirus disease 2019 in Korea. J Korean Med Sci. 2020;35(11):e124.

[26] Nwachukwu I, Nkire N, Shalaby R, et al. COVID-19 pandemic: age-related differences in measures of stress, anxiety and depression in Canada. Int J Environ Res Public Health. 2020;17(17):6366.

[27] Shaw K, Butcher S, Ko J, et al. Wearing of cloth or disposable surgical face masks has no effect on vigorous exercise performance in healthy individuals. Int J Environ Res Public Health. 2020;17(21):8110. 